\title{
Optimización Multiobjetivo del Proceso Fenton en el Tratamiento de Aguas Residuales provenientes de la Producción de Café Soluble
}

\author{
Harold N. Ibarra-Taquez', Izabela Dobrosz-Gómez² y Miguel-Ángel Gómez ${ }^{1}$ \\ (1) Departamento de Ingeniería Química, Facultad de Ingeniería y Arquitectura, Universidad Nacional de \\ Colombia, Sede Manizales, Colombia (e-mail: hnibarrat@unal.edu.co; magomez@unal.edu.co) \\ (2) Departamento de Física y Química, Facultad de Ciencias Exactas y Naturales, Universidad Nacional de \\ Colombia, Sede Manizales. Campus La Nubia, Apartado Aéreo 127, Manizales, Colombia \\ (e-mail: idobrosz-gomez@unal.edu.co;
}

Recibido Feb. 13, 2018; Aceptado May. 2, 2018; Versión final May. 30, 2018, Publicado Oct. 2018

\begin{abstract}
Resumen
Se evaluó el proceso Fenton en el tratamiento de las aguas residuales provenientes de una industria de café soluble colombiana. Se utilizó un diseño experimental del tipo Box-Behnken y la metodología de la superficie de respuesta para determinar los valores de los parámetros operativos que permiten alcanzar la más alta reducción de la demanda química de oxigeno (DQO) y/o del color. Los valores máximos alcanzados fueron de $80.5 \%$ y $85.3 \%$, respectivamente. Utilizando una evaluación económica y la metodología de la deseabilidad global, se encontraron las condiciones operativas ideales para cumplir tanto con la restricción ambiental de la DQO de la legislación colombiana como con los mínimos costos operacionales. Con base a los resultados del estudio, se pudo concluir que el proceso Fenton puede constituirse en una alternativa completa o configurarse como una etapa adicional dentro de un esquema de tratamiento de aguas residuales de la industria del café soluble.
\end{abstract}

\section{Multi-objective Optimization of the Fenton Process for the Treatment of Soluble Coffee Wastewater}

\begin{abstract}
The Fenton process for the treatment of wastewater coming from a soluble coffee industry in Colombia is evaluated. An experimental design of the Box-Behnken type and the response surface methodology were used to determine the values of operating parameters that allow achieving the highest reduction in chemical oxygen demand (COD) and/or color. The maximum values of COD and color reduction were $80.5 \%$ and $85.3 \%$, respectively. Using an economic evaluation and the methodology of global desirability, the ideal operating conditions were determined to comply with both the environmental COD restriction of Colombian legislation and with the minimum operational costs. Based on the results of this study, one can see that Fenton process can be considered as a complete alternative or as an additional stage within a wastewater treatment scheme for the soluble coffee industry.
\end{abstract}

Keywords: wastewater treatment; soluble coffee industry; Fenton process; optimization 


\section{INTRODUCCIÓN}

Se estima que el mercado mundial del café soluble alcanza anualmente transacciones por cerca de 28 mil millones de dólares y sus proyecciones muestran que estas crecerán hasta en 8.3 mil millones adicionales para el 2020 (Shoup, 2016). Entre los países participes de este mercado como exportadores, Colombia ocupa el sexto lugar con 800 mil sacos de café soluble exportados en el 2016 (Foreign Agricultural Service, 2017). La producción en este país tiende a crecer en el futuro debido a la calidad del grano y sus adecuados procesos de producción. Pese a los altos niveles técnicos utilizados en el proceso de elaboración del café soluble, éste genera una alta cantidad de aguas residuales. En la literatura se reporta que esta entre 40 y 45 litros de agua residual por kilogramo de café soluble producido (Oller et al., 2011), aunque estos valores dependen de las prácticas de ahorro, reutilización de agua y del tipo de extracción del café (Clarke y Vitzthum, 2001). Estas aguas residuales corresponden a una mezcla compleja de sustancias, conformada entre otras por ácidos clorogénicos, ácidos húmicos, polisacáridos y cafeína (Farah, 2012; Panchangam y Janakiraman, 2015).

Las aguas residuales del proceso de producción de café soluble se caracterizan por altos niveles de Demanda Química de Oxígeno (DQO) y un pH ácido. La magnitud del problema ambiental se incrementa debido a que las aguas poseen adicionalmente un intenso color oscuro, el cual puede interferir con la normal radiación solar hacia los organismos acuáticos y alterar la cadena trófica de cualquier cuerpo de agua si estas son vertidas sin un tratamiento previo. Para este tipo de aguas residuales, los tratamientos convencionales solo logran disminuir levemente las cargas contaminantes de color y DQO. Entre estos métodos se encuentran la coagulación química y floculación (Zayas et al., 2007) y los procesos biológicos en los que se incluyen reactores UASB (Dinsdale et al., 1997). En los casos reportados, los procesos de coagulación química y floculación han involucrado altas dosis de coagulantes. Por otro lado, los procesos biológicos han implicado altos tiempos de retención y, en algunas ocasiones, inestabilidad debido a la presencia de componentes inhibitorios para los microrganismos (Fernandez y Forster, 1993). De hecho, estas aguas residuales generalmente presentan una relación de Demanda Biológica de Oxígeno (DBO) a DQO menor que 0.4, el límite de biodegradabilidad.

Se han propuesto alternativas para enfrentar el reto de la decoloración y reducción de DQO de las aguas residuales provenientes de la industria de café soluble. Entre ellas se encuentran los Procesos Foto-Fenton asistidos por radiación UV (Tokumura et al., 2006) o energía solar (Tokumura et al., 2008). También se han utilizado procesos de coagulación-floculación acoplados a Procesos Avanzados de Oxidación (PAO) como por ejemplo UV/ $\mathrm{H}_{2} \mathrm{O}_{2}, \mathrm{UV} / \mathrm{O}_{3}$ o UV/ $\mathrm{H}_{2} \mathrm{O}_{2} / \mathrm{O}_{3}$ en condiciones ácidas (Zayas et al., 2007) o Procesos Avanzados de Oxidación Electroquímicos (PAOE) usando Ánodos Dimensionalmente Estables (ADE) (Cárdenas et al., 2009) o usando electrodos de Diamante Dopado con Boro (DDB) (Villanueva-Rodríguez et al., 2014). Muchas de estas tecnologías solo han sido evaluadas a pequeña escala y los costos de capital implicados en su implementación continúan siendo demasiado altos. Otro tratamiento alternativo incluyó un esquema secuencial de procesos electroquímicos usando electrodos de aluminio y grafito (Ibarra-Taquez et al., 2017). En él se usó una primera etapa de electrocoagulación para reducir el color y se acopló una etapa posterior de electroxidación para reducir la concentración de DQO hasta alcanzar los límites impuestos por la legislación colombiana (que corresponde a $1000 \mathrm{mg} / \mathrm{l}$ según el Ministerio de Ambiente y Desarrollo Sostenible de Colombia, 2015). Sin embargo, existen tratamientos menos demandantes energéticamente como el proceso Fenton cuyo desempeño en el tratamiento de aguas residuales complejas ha demostrado ser eficiente (Kušić et al., 2007; Bianco et al., 2011; Prazeres et al., 2013).

El proceso Fenton tiene varias ventajas competitivas, como su diseño simple, su funcionamiento no promueve la generación de compuestos órgano-clorados, los reactivos usados son de amplia disposición, de fácil almacenamiento y manejo y sus posibles remanentes son inocuos para el medio ambiente (Babuponnusami y Muthukumar, 2014; Wang et al., 2016). Su operación consiste en la generación de radicales hidroxilo capaces de oxidar la materia orgánica y destruir muchos de los componentes recalcitrantes o tóxicos en el agua residual a partir de iones ferrosos y peróxido de hidrógeno (Bagal y Gogate, 2014) (ecuación (1)):

$$
\mathrm{Fe}^{2+}+\mathrm{H}_{2} \mathrm{O}_{2} \rightarrow \mathrm{Fe}^{3+}+\mathrm{OH} \cdot+\mathrm{OH}^{-}
$$

El ion férrico puede descomponer al peróxido de hidrógeno en agua y en oxígeno formando nuevamente el ion ferroso y el radical hidroperoxilo, de menor poder oxidante que el radical hidroxilo (ecuaciones (2) y (3)):

$$
\begin{aligned}
& \mathrm{Fe}^{3+}+\mathrm{H}_{2} \mathrm{O}_{2} \leftrightarrow \mathrm{Fe}-\mathrm{OOH}^{2+}+\mathrm{H}^{+} \\
& \mathrm{Fe}-\mathrm{OOH}^{2+} \rightarrow \mathrm{HO}_{2} \cdot+\mathrm{Fe}^{2+}
\end{aligned}
$$


La eficiencia del proceso Fenton depende básicamente del contenido de materia orgánica del agua residual, la temperatura, la relación $\mathrm{Fe}^{2+}$ a peróxido, la concentración de peróxido y el pH (Guedes et al., 2003). Pese a que el proceso Fenton es endotérmico. Así, en la mayoría de casos aplicados a la industria se utilizan las condiciones medio ambientales para su operación, evitando así gastos energéticos adicionales. Las condiciones de operación dependen del efluente a tratar y deben ser optimizadas para cada caso particular. Por estas razones, en este trabajo se evalúa el desempeño del proceso Fenton para el tratamiento de las aguas residuales provenientes de la industria de café soluble. Se analiza la influencia de tres factores operacionales: $\mathrm{pH}$ inicial, concentración de hierro y concentración de peróxido. El trabajo involucra un diseño factorial tipo Box-Behnken junto con la metodología de la superficie de respuesta para optimizar la eficiencia del proceso. Estas herramientas han probado ser útiles para la caracterización de sistemas de tratamiento utilizando Procesos Avanzados de Oxidación (PAOs) (Ay et al., 2009; Bianco et al., 2011; GilPavas et al., 2016a). También se incluye un análisis de costos operacionales para encontrar condiciones adecuadas de operación utilizando la metodología de la función de deseabilidad global.

\section{METODOLOGÍA}

La matriz utilizada para desarrollar los diferentes ensayos fue una muestra representativa de las aguas residuales de una industria de café soluble, localizada en la región cafetera de Colombia. Sus características se representan en la Tabla 1. La DQO de esta agua residual es tres veces más alta que el límite permitido en Colombia (Ministerio de Ambiente y Desarrollo Sostenible de Colombia, 2015). Su alto valor implica la presencia de gran cantidad de materia orgánica. Además, la relación inicial $\mathrm{DBO}_{5} / \mathrm{DQO}$ sugiere que el efluente analizado no es susceptible a la biodegradación.

Tabla 1: Características del agua residual proveniente de una industria de café soluble ubicada en la región andina colombiana

\begin{tabular}{|l|l|c|c|}
\hline Parámetro & Unidades & Agua residual & Valor máximo permitido \\
\hline Conductividad & $\mu \mathrm{S} / \mathrm{cm}$ & 465 & No reporta \\
\hline Turbidez & $\mathrm{NTU}$ & 12 & No reporta \\
\hline $\mathrm{pH}$ & $(-)$ & 4.7 & $6.0-9.0$ \\
\hline Demanda Química de Oxígeno (DQO) & $\mathrm{mg} / \mathrm{l}$ & 3528 & 1000 \\
\hline Demanda Biológica de Oxígeno (DBO5) & $\mathrm{mg} / \mathrm{l}$ & 1267 & 600 \\
\hline Carbón Orgánico Total (COT) & $\mathrm{mg} / \mathrm{l}$ & 1314 & No reporta \\
\hline Color & $\mathrm{U} \mathrm{Pt}-\mathrm{Co}$ & 3046 & Análisis y reporte \\
\hline Solidos suspendidos totales & $\mathrm{mg} / \mathrm{l}$ & 21 & 400 \\
\hline
\end{tabular}

El agua residual a tratar se dispuso en un recipiente de $200 \mathrm{ml}$, a temperatura ambiente controlada $\left(20^{\circ} \mathrm{C}\right)$. Se ajustó su pH a un valor específico utilizando una solución 3 molar de ácido sulfúrico (95-97\%, Merck). Para dar inicio a la reacción de Fenton, el peróxido de hidrógeno se agregó a partir de una solución $30 \% \mathrm{~m} / \mathrm{m}$ (Sigma-Aldrich) y luego la sal de hierro a partir de una solución de 10000 ppm de hierro preparada con sulfato ferroso heptahidratado $\left(\mathrm{FeSO}_{4} .7 \mathrm{H}_{2} \mathrm{O}\right.$, grado extrapuro, Duksan). Con agitación constante de $350 \mathrm{RPM}$, el proceso se llevó a cabo durante tres horas al final de las cuales se tomaron muestras para mediciones de DQO, color y peróxido remanente. Para determinar la concentración de DQO se utilizó el método de reflujo cerrado con mediciones espectro-fotométricas reportado en el método estándar 5220D (APHA, 2005) mientras que las mediciones cuantitativas de color se llevaron a cabo a partir del método estándar $2120 \mathrm{C}$ (APHA, 2005). La determinación de la concentración de peróxido remanente se realizó mediante titulación con permanganato de potasio (USP Technologies, 2017).

Se estudió la influencia del pH inicial $\left(X_{1}\right)$, la concentración de hierro $\left(X_{2}\right)$ y la concentración de peróxido $\left(X_{3}\right)$ durante el proceso Fenton por medio de un Diseño experimental Box-Behnken (DBB). El DBB se basa en un diseño factorial de tres niveles incompleto con características de segundo orden rotable y fue seleccionado por su eficiencia y bajo número de ensayos experimentales para determinar la significancia estadística de los factores (Ferreira et al., 2007; Gilpavas et al., 2016b). El DBB con tres factores involucró 12 pruebas. Adicionalmente, para evaluar el error experimental y aumentar los grados de libertad, se incluyeron cuatro puntos centrales obteniendo así un diseño experimental con un total de 16 ensayos. Todos los experimentos se desarrollaron por triplicado. El orden de los experimentos se realizó de forma aleatoria con la ayuda del software estadístico Statgraphics Centurión XVI para evitar el efecto de variables escondidas. El rango de estudio para cada factor fue establecido basándose en experimentos preliminares (no incluidos aquí) de forma que a través del proceso Fenton se consiguiera algún nivel de reducción de contaminantes. Como variables 
de respuesta para el análisis del desempeño del proceso Fenton se seleccionaron la reducción de color y de DQO definidas según las ecuaciones (4) y (5):

$$
\begin{aligned}
& R_{\text {color }}=\frac{\left(C_{\text {color }}^{0}-C_{\text {color }}^{\text {fin }}\right)}{C_{\text {color }}^{0}} \\
& R_{D Q O}=\frac{\left(C_{D Q O}^{0}-C_{D Q O}^{\text {fin }}\right)}{C_{D Q O}^{0}}
\end{aligned}
$$

Donde $\mathrm{C}_{\text {color }} \mathrm{y}_{\mathrm{DQO}}$ son las concentraciones de color y de $\mathrm{DQO}$ en unidades de $\mathrm{U} \mathrm{Pt}$-Co y $\mathrm{mg} / \mathrm{l}$, respectivamente. Los superíndices 0 y fin corresponden a las concentraciones al inicio y al final del tratamiento.

Posteriormente se evaluó estadísticamente la relación entre una función polinómica de segundo orden y las variables independientes basándose en un análisis de varianza (ANOVA). Esta función polinómica puede ser descrita por la forma general presentada en la ecuación (6):

$$
Y_{i}=\beta_{0}+\sum_{i=1}^{3} \beta_{i} X_{i}+\sum_{i=1}^{3} \beta_{i i} X_{i}^{2}+\sum_{i=1}^{3} \sum_{j=1}^{3} \beta_{i j} X_{i} X_{j}
$$

Donde $Y_{i}$ es cualquiera de las dos variables de respuesta establecidas, $\mathrm{Xi}$ y $\mathrm{Xj}$ son los factores experimentales, $\beta_{0}$ es el coeficiente del intercepto, $\beta_{i}$ es el coeficiente lineal para el factor $\mathrm{i}, \beta_{\mathrm{ij}}$ es el coeficiente cuadrático para su factor i correspondiente y $\beta_{\mathrm{ij}}$ es el coeficiente de interacción entre el factor i y j. Estos coeficientes fueron ajustados a través del software estadístico utilizando los resultados obtenidos en el diseño experimental. A través del análisis estadístico se construyeron también diagramas de Pareto. El modelo polinómico adicionalmente permitió optimizar la reducción de DQO o la reducción de color a través del método de la superficie de respuesta.

Los costos operacionales del proceso Fenton se calcularon en términos de dólares americanos (USD) por metro cúbico de agua tratada, teniendo en cuenta el consumo de sulfato de hierro y peróxido de hidrógeno. Sus precios en el mercado colombiano para el año 2017, teniendo en cuenta los índices de costos reportados por el Departamento Administrativo Nacional de Estadística (DANE), fueron de 108 USD/ton para el sulfato de hierro heptahidratado con una pureza del $98 \%$ y de 456 USD/ton para el peróxido de hidrógeno a una concentración de $35 \% \mathrm{~m} / \mathrm{m}$. Con el propósito de sintetizar métodos de tratamiento con bajos costos operacionales, una destacada reducción de DQO y/o de reducción de color este trabajo se complementó con una serie de optimizaciones de múltiples respuestas utilizando el método de la función de deseabilidad (Colombo et al., 2013). Este método consiste inicialmente en transformar cada variable de respuesta en una función de deseabilidad, $d_{i}$, cuyos valores van de 0 a 1 , que representan la condición de menor y mayor favorabilidad para el proceso, respectivamente. Las funciones de deseabilidad individual resultantes para el proceso Fenton se muestran en las ecuaciones (7)-(9):

$$
\begin{aligned}
& d_{1}(X)=\left[\frac{Y_{1}(X)-E l_{1}}{E S_{1}-E l_{1}}\right]^{s_{1}} \\
& d_{2}(X)=\left\{\begin{array}{cl}
{\left[\frac{Y_{2}(X)-E I_{2}}{T-E I_{2}}\right]^{S_{2}},} & , E_{2} \leq Y_{2}(X) \leq T_{2} \\
1, & , T<Y_{2}(X) \leq E S_{2}
\end{array}\right. \\
& d_{3}(X)=\left[\frac{E_{3}-Y_{3}(X)}{E S_{3}-E I_{3}}\right]^{s_{3}}
\end{aligned}
$$

Donde $Y_{1}$ y $Y_{2}$ representan la $R_{\text {Color }}$ y la $R_{D Q O}$, respectivamente. Se incluyó como variable de respuesta para este método la variable $Y_{3}$ correspondiente a los costos operacionales totales; $E_{i}$ y $E_{i}$ son los valores inferiores y superiores que puede tener la i-ésima variable de respuesta, según el intervalo experimental estudiado. Su inclusión dentro de cada función de deseabilidad es necesaria para normalizar su rango entre 
0 y 1; T es el valor objetivo o nominal para la reducción de DQO (Y2 objetivo), que para el caso de estudio corresponde a 0.7166 y que garantiza alcanzar la concentración límite exigida por la legislación ambiental colombiana. Dado que no existe un límite impuesto por la legislación ambiental colombiana relacionado con el color de las aguas residuales estudiadas y el costo operacional tampoco está restringido, las funciones $d_{1}$ $\mathrm{y}_{3}$ no poseen un valor objetivo $T$. Nótese adicionalmente que estas funciones de deseabilidad cambian de una a la otra porque el propósito para las dos primeras funciones es maximizar las reducciones mientras que para la tercera función el objetivo es minimizar los costos. El exponente $s_{i}$ permite elegir la forma de la función transformada. Cuando $s_{\mathrm{i}}$ es 1 la función de deseabilidad individual tiene el mismo comportamiento del modelo original. Un valor mayor a 1 refleja un mayor interés en estar cerca del objetivo mientras que un valor menor a 1 le da menor importancia a los cambios que se generan cerca del objetivo (Montgomery, 2012). Para que el tipo de optimización se transforme en encontrar el máximo de una única función, finalmente se define la función de deseabilidad usando la media geométrica ponderada de las deseabilidades individuales y factores de peso (ecuación (10)):

$$
\mathrm{DG}(\mathrm{X})=\left(\mathrm{d}_{1}^{\mathrm{w}_{1}} \times \mathrm{d}_{2}^{\mathrm{w}_{2}} \times \mathrm{d}_{3}^{\mathrm{w}_{3}}\right)^{\frac{1}{\sum \mathrm{w}_{\mathrm{i}}}}
$$

Los factores de peso $w_{i}$ están relacionados directamente con la importancia relativa de cada respuesta en el proceso y sus valores serán analizados en la sección de resultados para considerar diferentes configuraciones del proceso. Para facilitar el análisis se definió que $\sum \mathrm{w}_{\mathrm{i}}=1$.

\section{RESULTADOS Y DISCUSIÓN}

En la Tabla 2 se presenta la matriz correspondiente al DBB. En ella se puede observar, entre otros aspectos, el intervalo experimental utilizado para el $\mathrm{pH}$, la concentración de hierro, $\mathrm{C}_{\mathrm{Fe}}$, y la concentración de peróxido, $\mathrm{C}_{\mathrm{H}_{2} \mathrm{O}_{2}}$. Las pruebas se muestran ordenadas de forma ascendente pero en su ejecución el orden fue aleatorio.

Tabla 2: Matriz del diseño experimental y resultados de las variables de respuesta

\begin{tabular}{|c|c|c|c|c|c|}
\hline Ensayo & $\begin{array}{c}\mathrm{pH} \\
\left(\mathrm{X}_{1}\right)\end{array}$ & $\begin{array}{c}\mathrm{CFe}_{\mathrm{Fe}}[\mathrm{mg} / \mathrm{L}] \\
\left(\mathrm{X}_{2}\right)\end{array}$ & $\begin{array}{c}\mathrm{C}_{\mathrm{H} 2 \mathrm{O} 2}[\mathrm{mg} / \mathrm{L}] \\
\left(\mathrm{X}_{3}\right)\end{array}$ & $\begin{array}{c}\text { Reducción de color } \\
\left(\mathrm{Y}_{1}\right)\end{array}$ & $\begin{array}{c}\text { Reducción de DQO } \\
\left(\mathrm{Y}_{2}\right)\end{array}$ \\
\hline 1 & 2.5 & 100 & 4500 & $74.08 \%$ & $58.39 \%$ \\
\hline 2 & 2.5 & 300 & 3000 & $47.60 \%$ & $47.88 \%$ \\
\hline 3 & 2.5 & 300 & 6000 & $84.16 \%$ & $75.83 \%$ \\
\hline 4 & 2.5 & 500 & 4500 & $66.56 \%$ & $65.57 \%$ \\
\hline 5 & 3.6 & 100 & 3000 & $58.90 \%$ & $53.98 \%$ \\
\hline 6 & 3.6 & 100 & 6000 & $73.60 \%$ & $52.93 \%$ \\
\hline 7 & 3.6 & 300 & 4500 & $71.03 \%$ & $64.28 \%$ \\
\hline 8 & 3.6 & 300 & 4500 & $67.44 \%$ & $65.11 \%$ \\
\hline 9 & 3.6 & 300 & 4500 & $70.92 \%$ & $66.03 \%$ \\
\hline 10 & 3.6 & 300 & 4500 & $74.64 \%$ & $65.38 \%$ \\
\hline 11 & 3.6 & 500 & 3000 & $36.35 \%$ & $51.01 \%$ \\
\hline 12 & 3.6 & 500 & 6000 & $70.27 \%$ & $74.67 \%$ \\
\hline 13 & 4.7 & 100 & 4500 & $42.74 \%$ & $35.30 \%$ \\
\hline 14 & 4.7 & 300 & 3000 & $40.12 \%$ & $48.19 \%$ \\
\hline 15 & 4.7 & 300 & 6000 & $80.78 \%$ & $71.36 \%$ \\
\hline 16 & 4.7 & 500 & 4500 & $53.12 \%$ & $64.61 \%$ \\
\hline
\end{tabular}

La más alta reducción de contaminantes se alcanzó en el ensayo 3, con la mayor concentración de peróxido y una concentración de hierro intermedia. Esta relación proporciona una alta disposición de reactivos para la generación de radical hidroxilo proveniente de la reacción descrita en la ecuación (1). En este ensayo el pH es altamente ácido, condición favorable para el proceso Fenton (v.g., implica una baja precipitación de hierro y alta generación de especies activas) (Pignatello et al., 2006). Una alta reducción de DQO también se puede observar en el ensayo 12, sin embargo la reducción de color es baja posiblemente por la formación de complejos químicos entre el hierro y los componentes de las aguas del café soluble. 


\section{Análisis estadístico y modelos polinómicos}

Los datos recolectados en el diseño experimental se correlacionaron, para cada una de las variables de respuesta, mediante modelos polinómicos de segundo orden. Se obtuvieron las expresiones presentadas en las ecuaciones (11) y (12):

$$
\begin{aligned}
& \mathrm{Y}_{1}=\mathrm{R}_{\text {Color }}=0.09522+0.1006 \times \mathrm{pH}-4.518 \times 10^{-4} \times \mathrm{C}_{\mathrm{Fe}}+1.782 \times 10^{-4} \times \mathrm{C}_{\mathrm{H}_{2} \mathrm{O}_{2}}-0.03511 \times \mathrm{pH}^{2}+ \\
& 2.034 \times 10^{-4} \times \mathrm{pH} \times \mathrm{C}_{\mathrm{F}}+6.211 \times 10^{-6} \times \mathrm{pH} \times \mathrm{C}_{\mathrm{H}_{2} \mathrm{O}_{2}}{ }^{-1.908 \times 10^{-6}} \times \mathrm{C}_{\mathrm{Fe}}{ }^{2}+1.602 \times 10^{-7} \times \mathrm{C}_{\mathrm{Fe}} \times \mathrm{C}_{\mathrm{H}_{2} \mathrm{O}_{2}}{ }^{-} \\
& 1.597 \times 10^{-8} \times \mathrm{C}_{\mathrm{H}_{2} \mathrm{O}_{2}}{ }^{2} \\
& \mathrm{Y}_{2}=\mathrm{R}_{\mathrm{DQO}}=0.2350+0.1203 \times \mathrm{pH}-5.94 \times 10^{-4} \times \mathrm{C}_{\mathrm{Fe}}+6.986 \times 10^{-5} \times \mathrm{C}_{\mathrm{H}_{2} \mathrm{O}_{2}}-0.02712 \times \mathrm{pH}^{2}+
\end{aligned}
$$

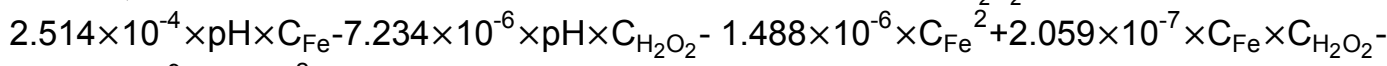

$$
\begin{aligned}
& 4.905 \times 10^{-9} \times \mathrm{C}_{\mathrm{H}_{2} \mathrm{O}_{2}}{ }^{2}
\end{aligned}
$$

El análisis estadístico de los modelos utilizando el test de Fisher para el ANOVA permitió obtener los resultados mostrados en la Tabla 3 y 4 para la reducción de color y DQO, respectivamente. De esta forma se puede establecer, por el alto valor del valor $F$ y el bajo valor de $p(<0.05)$ para ambos modelos, que la mayoría de las variaciones en las respuestas pueden explicarse a través de las ecuaciones ajustadas y que las variables de los modelos son estadísticamente significativas para esas respuestas con un alto nivel de probabilidad. Adicionalmente, se presentan en las tablas los coeficientes de correlación, $\mathrm{R}^{2}$, los cuales

\begin{tabular}{|c|c|c|c|c|c|}
\hline Fuente & $\begin{array}{c}\text { Suma de } \\
\text { Cuadrados }\end{array}$ & $\mathrm{GL}$ & $\begin{array}{c}\text { Cuadrado } \\
\text { Medio }\end{array}$ & Razón-F & Valor-p \\
\hline pH: Término lineal + Cúbico & 0.04592 & 2 & 0.0230 & 3.671 & 0.0909 \\
\hline Fe: Término lineal + Cúbico & 0.02993 & 2 & 0.0150 & 2.393 & 0.1721 \\
\hline $\mathrm{H}_{2} \mathrm{O}_{2}$ : Término lineal + Cúbico & 0.2031 & 2 & 0.1016 & 16.24 & 0.0038 \\
\hline Interacciones & 0.01767 & 3 & 0.0059 & 0.9416 & 0.4772 \\
\hline Modelo & 0.2966 & 9 & 0.0330 & 5.2700 & 0.0280 \\
\hline & $\mathrm{R}^{2}$ & 0.8877 & \multicolumn{2}{|c|}{$\mathrm{R}^{2}$ ajustado: } & 0.7193 \\
\hline
\end{tabular}
expresan una buena correlación entre los valores observados y predichos, teniendo en cuenta que su valor puede ser considerado como aceptable si $\mathrm{R}^{2}$ es mayor a 0.7 (Gutierrez-Pulido y De la Vara, 2008).

\begin{tabular}{|c|c|c|c|c|c|}
\hline Fuente & $\begin{array}{l}\text { Suma de } \\
\text { Cuadrados }\end{array}$ & $\mathrm{GL}$ & $\begin{array}{c}\text { Cuadrado } \\
\text { Medio }\end{array}$ & Razón-F & Valor-p \\
\hline pH: Término lineal + Cúbico & 0.01426 & 2 & 0.0071 & 2.230 & 0.1887 \\
\hline Fe: Término lineal + Cúbico & 0.05232 & 2 & 0.0262 & 8.179 & 0.0193 \\
\hline $\mathrm{H}_{2} \mathrm{O}_{2:}$ Término lineal + Cúbico & 0.06845 & 2 & 0.0342 & 10.70 & 0.0105 \\
\hline Interacciones & 0.02810 & 3 & 0.0094 & 2.926 & 0.1219 \\
\hline Modelo & 0.1631 & 9 & 0.0181 & 5.667 & 0.0235 \\
\hline & $\mathrm{R}^{2}$ : & 0.8947 & \multicolumn{2}{|c|}{$\mathrm{R}^{2}$ ajustado: } & 0.7368 \\
\hline
\end{tabular}

Tabla 3: Análisis de varianza (ANOVA) para el modelo polinómico de reducción de color en el proceso Fenton

Tabla 4: Análisis de varianza (ANOVA) para el modelo polinómico de reducción de DQO en el proceso Fenton

De los diagramas de Pareto presentados en la Fig. 1 se puede observar que las interacciones entre las variables del modelo no tienen efecto significativo sobre las variables de respuesta. Este hecho es evidente si se tiene en cuenta que toda variable que sobrepase la línea punteada puede considerarse estadísticamente significativa con un nivel de confianza del 95\% según una distribución t de student. En los diagramas de Pareto también se incluye el cálculo del porcentaje de importancia relativa a cada factor $\left(P F_{i}\right)$ calculado a partir de la ecuación (13):

$$
P F_{i}=\left(\frac{b_{i}^{2}}{\sum b_{i}^{2}}\right) \times 100 \quad(i \neq 0)
$$


Donde $b_{i}$ corresponde al valor del efecto estandarizado para el factor i representado por cada barra en el diagrama de Pareto. Nótese que la variable más significativa es la concentración de peróxido y que su influencia es directamente proporcional a la reducción tanto del color como de la DQO (ca. $67 \%$ y $42 \%$, respectivamente). Para ambas reducciones de contaminante solo una variable adicional tiene significancia estadística. En el caso de la reducción de color, ésta es el pH (ca. 13\%) mientras que para la reducción de DQO corresponde a la concentración de hierro (ca. 23\%). Se podría concluir también que, por su bajo efecto (v.g., <2\%), se podría prescindir de al menos dos de las interacciones consideradas en el modelo. Esto haría que el valor de $R^{2}$ ajustado incremente su valor y que sea más similar a $R^{2}$. Sin embargo, el valor de $R^{2}$ también disminuiría y haría las predicciones menos acertadas. Por esta razón para los cálculos posteriores se tuvieron en cuenta todos los efectos considerados inicialmente en los modelos.

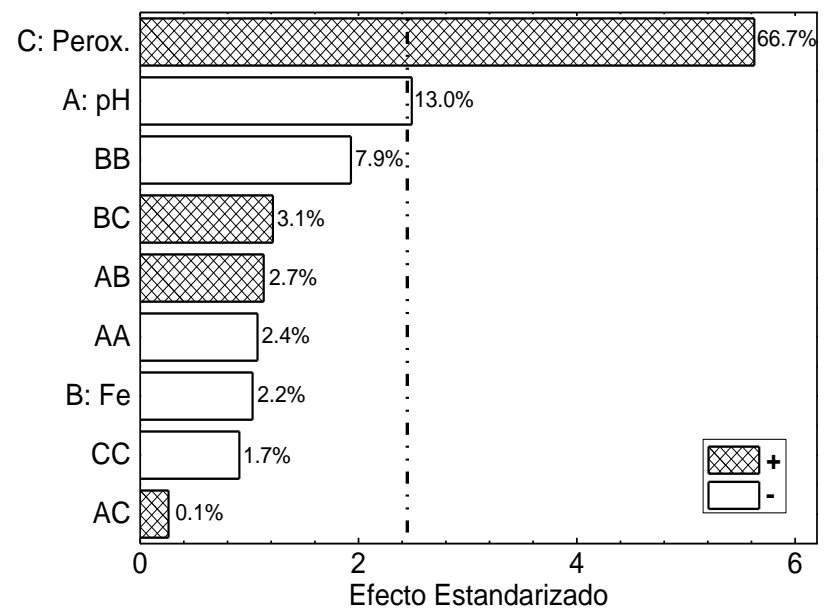

a)

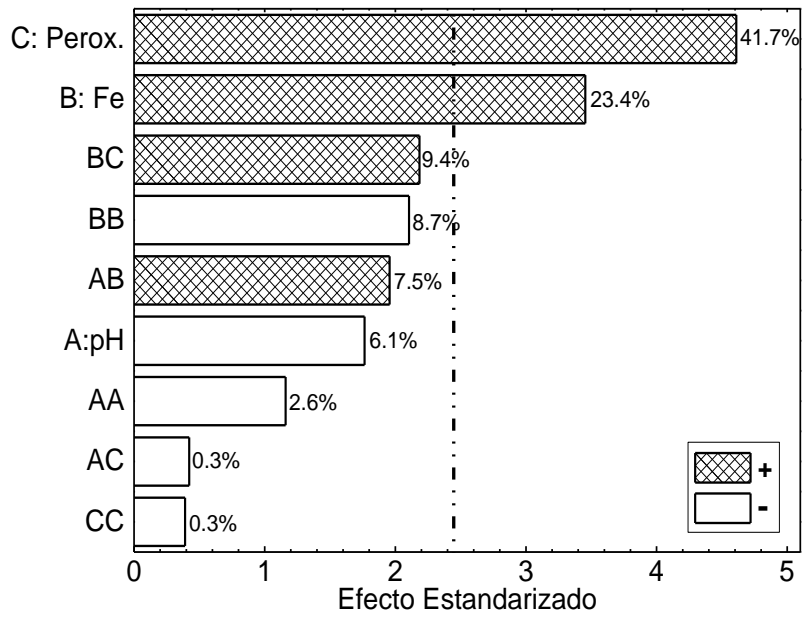

b)

Fig. 1: Diagramas de Pareto para: a) la reducción de color y b) la reducción de DQO

\section{Superficies de respuesta y optimización}

Con los polinomios ajustados se construyeron las superficies de respuesta para observar claramente la relación entre los factores, las reducciones de contaminante y la presencia de condiciones óptimas (Fig. 2). De acuerdo con las superficies, las más altas reducciones se obtienen con la mayor concentración de peróxido. Por otro lado, la concentración de hierro también favorece la reducción de DQO a concentraciones altas de peróxido mientras que existe una condición óptima de hierro para la reducción de color. Esto se debe al hecho mencionado previamente por el cual a altas concentraciones de hierro las aguas residuales pueden formar complejos (que intensifican color oscuro del agua residual). Sin embargo, las reacciones Fenton no son completamente efectivas si la concentración de hierro es baja.

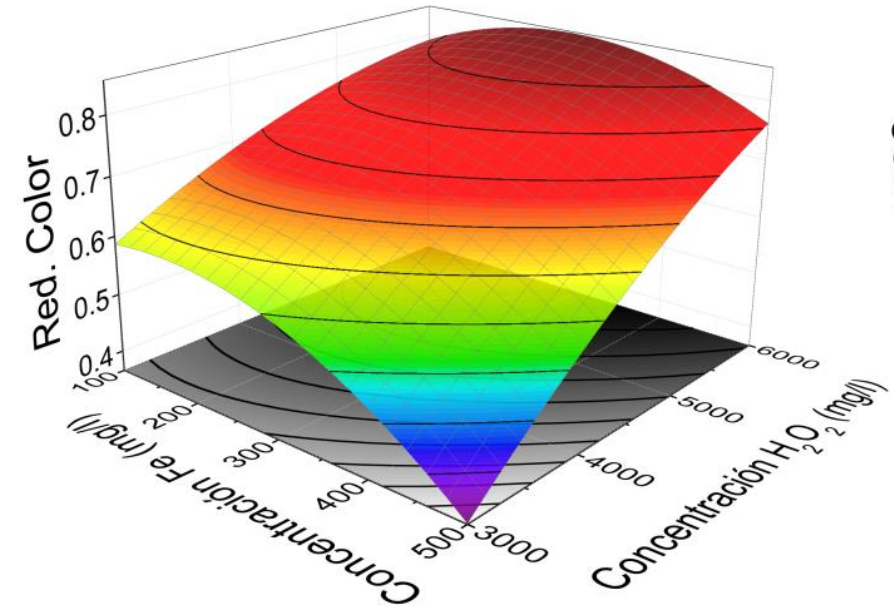

a)

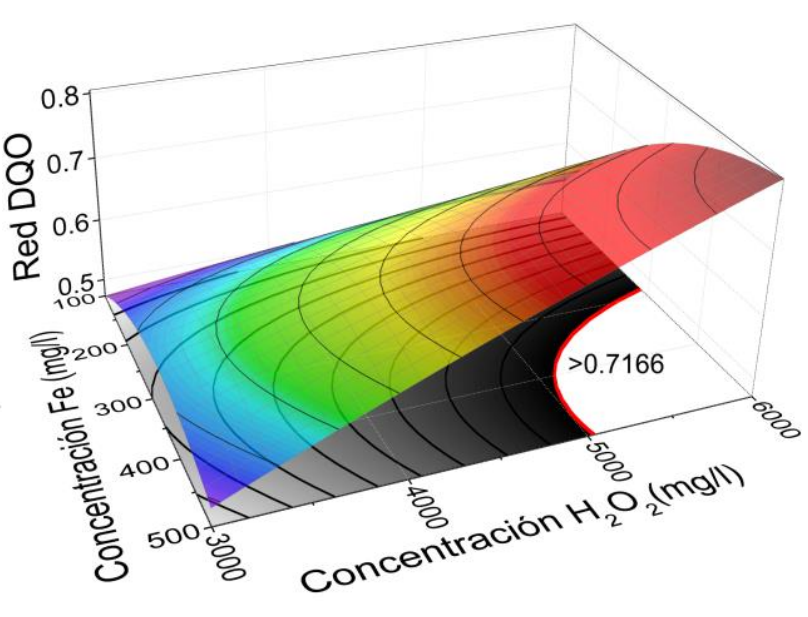

b)

Fig. 2: Superficies de respuesta para: a) la reducción de color y b) la reducción de DQO en sus pH óptimos (2.8 y 3.7)

En el contorno de la superficie de respuesta correspondiente a la reducción de DQO se puede observar una zona donde la reducción de DQO es mayor a ca. $72 \%$ (Fig. 2a). Esta zona puede ser considerada como la 
zona de operación permisible si se tiene en cuenta que bajo las condiciones de operación que la enmarcan se puede alcanzar los niveles de DQO exigidos por la legislación ambiental colombiana. Sin embargo, como se puede observar en la segunda superficie de respuesta (Fig. 2b), no en toda esa zona la reducción de color es alta. En la Tabla 5 se presentan las condiciones correspondientes a los valores más altos de reducciones de contaminante para el intervalo experimental analizado. Nótese que la concentración de peróxido más alta considerada en el diseño permite las más altas reducciones. Sin embargo, el óptimo respecto a la concentración de hierro y $\mathrm{pH}$ se encuentra a diferentes condiciones. Para verificar la capacidad predictiva de los modelos propuestos, se evaluó experimentalmente el proceso Fenton bajo las condiciones óptimas correspondientes a la máxima reducción de DQO. Se determinó que esa reducción fue igual a $80.86 \%$ mientras que la reducción de color fue de 70.44 \% (Tabla 5). Su comparación con los resultados predichos corrobora la buena capacidad del modelo para el cálculo de la reducción de DQO pero evidencia una predicción superior en la reducción de color. Esto posiblemente es causado por la complejidad de las interacciones entre el hierro y las sustancias fenólicas que aportan color a la solución reportada por algunos autores en los procesos Fenton (Adhoum y Monser, 2004; Tokumura et al., 2006).

Tabla 5: Condiciones óptimas de las variables de respuesta dentro del rango del diseño experimental

\begin{tabular}{|c|c|c|c|c|c|c|}
\hline & \multicolumn{3}{|c|}{ Condiciones del óptimo } & \multicolumn{3}{|c|}{$\begin{array}{c}\text { Valores de las variables de respuesta a } \\
\text { condiciones óptimas }\end{array}$} \\
\hline Parámetro & $\mathrm{pH}$ & $\begin{array}{c}\mathrm{Fe} \\
(\mathrm{mg} / \mathrm{l})\end{array}$ & $\begin{array}{l}\mathrm{H}_{2} \mathrm{O}_{2} \\
(\mathrm{mg} / \mathrm{l})\end{array}$ & Red. DQO & Red. Color & $\begin{array}{l}\text { Costo OP. } \\
\left(\text { USD } / \mathrm{m}^{3}\right)\end{array}$ \\
\hline Red. DQO & 3.73 & 500 & 6000 & $80.50 \%$ & $77.19 \%$ & 8.114 \\
\hline Red. Color & 2.78 & 281.5 & 6000 & $74.13 \%$ & $85.27 \%$ & 7.984 \\
\hline $\begin{array}{c}\text { Costo } \\
\text { Operacional }\end{array}$ & -- & 100 & 3000 & $47.16 \%$ & $50.10 \%$ & 3.968 \\
\hline
\end{tabular}

Al análisis de superficie de respuesta se complementó con la evaluación de los costos operacionales (C.OP). Considerando los precios y grado de purezas presentados en la sección de metodología, se evaluaron tales costos a partir de la ecuación (14) como función de la concentración de hierro y peróxido:

$$
\mathrm{Y}_{3}=\mathrm{C} . \mathrm{OP}\left(\frac{\mathrm{USD}}{\mathrm{m}^{3}}\right)=5.9273 \times 10^{-4} \times \mathrm{C}_{\mathrm{Fe}}+1.3029 \times 10^{-3} \times \mathrm{C}_{\mathrm{H}_{2} \mathrm{O}_{2}}
$$

Según la forma lineal de la expresión de los C.OP, evidentemente los costos aumentan con el incremento en las concentraciones de reactivos. De allí que las condiciones óptimas para esta variable se encuentran en el extremo inferior del rango experimental (Tabla 5). Para la evaluación de costos operacionales, el costo operacional implicado en el ajuste de $\mathrm{pH}$ no se tuvo en cuenta por representar un pequeño porcentaje frente al consumo de los otros reactivos. A las condiciones que permiten alcanzar los más bajos costos operacionales se obtienen simultáneamente las menores reducciones de DQO. Por lo tanto, el método de la superficie de respuesta no es posible evidenciar unas condiciones de operación que permitan alcanzar los valores exigidos por la legislación ambiental colombiana y obtener una alta decoloración con unos bajos costos operacionales. Por esto, se complementó en análisis usando el método de la función de deseabilidad global. Para este método se analizaron cinco optimizaciones, asignando diferentes factores de peso (Tabla 6). Cada optimización se puede observar como opciones de operación según los objetivos del tratamiento o como proyecciones hacia un acople óptimo con otras tecnologías. Para cada optimización se utilizó el valor de 2 en los exponentes $s$ con el objetivo de tener similares influencias de los factores frente a la deseabilidad global. En la Tabla 6 no se incluyeron los casos cuando el factor de peso es igual a 1 para una respuesta y cero en las restantes ya que esas condiciones corresponden a los resultados obtenidos en la optimización con el método de la superficie de respuesta y reportados en la Tabla 5.

El comportamiento de la concentración de peróxido y de hierro en la deseabilidad global se muestra en la Fig. 3 para el caso 1. Para observar claramente el punto de máxima deseabilidad global, la figura se realizó a su $\mathrm{pH}$ óptimo correspondiente a 2.7. Ese valor de máxima deseabilidad es cercano a 0.5 y los valores de los factores donde se encuentra se reportan en la Tabla 6. En esa tabla se presentan también los valores de reducción de DQO, de color y de costos operacionales. Bajo las condiciones óptimas, los costos son bajos pero no se alcanza el valor objetivo para la reducción de DQO. Este caso podría ser considerado cuando se acopla en una etapa posterior al proceso Fenton una tecnología de tratamiento económica y eficiente que permita alcanzar la reducción de DQO exigida por la legislación colombiana. Bajo las condiciones establecidas se garantizaría que la etapa correspondiente al proceso Fenton tenga una alta reducción de los contaminantes y un costo operacional bajo. En el caso 2, las variables que se seleccionaron con peso para optimizar fueron la reducción de color y los costos operacionales mientras que en el caso 3 se cambió la reducción de color 
por la reducción de DQO. En sus condiciones óptimas ninguno de estos dos casos permite cumplir la reducción de DQO requerida por la legislación colombiana y ambos casos deben ser complementados con otras tecnologías. Sin embargo, son procesos económicos y con buen desempeño para la reducción de color - DQO. Sus costos operacionales incluso son cercanos a la mitad de los generados bajo el óptimo de reducción de DQO mencionado en la Tabla 5. Se podría seleccionar las condiciones de operación del caso 2 si la tecnología complementaria es eficiente para la reducción de color más que para reducir la concentración de DQO o el caso 3 si la tecnología es más eficiente para la reducción de DQO. En el caso 4 se seleccionó como principal objetivo una alta reducción de DQO y de color sin importar los costos operativos. Bajo estas condiciones se alcanzan los valores exigidos por la legislación colombiana pero a costos elevados. Por esta razón en el caso 5 se varía el valor del peso correspondiente a los costos operacionales de tal forma que permita conseguir los más bajos costos operacionales pero sujeto a cumplir los requisitos de la legislación ambiental colombiana y a obtener alta reducción de color. Esto se consigue con un factor de peso igual a 0.1285 en los costos operacionales $\left(w_{3}{ }^{*}=0.1285\right)$. Debido a que los casos 4 y 5 ya cumplen la norma colombiana, ambas condiciones de operación podrían utilizarse cuando se considera que el color remanente no puede afectar la fuente hídrica en donde se descargará el efluente tratado (v.g., aguas naturalmente oscuras) o ante la imposibilidad de construir unidades adicionales de tratamiento.

Tabla 6: Optimización de la deseabilidad global con diferentes valores para los factores de peso

\begin{tabular}{|c|c|c|c|c|c|c|c|c|c|c|}
\hline \multirow{2}{*}{ caso } & \multicolumn{3}{|c|}{ Factores de peso } & \multicolumn{3}{c|}{ Condiciones del óptimo } & \multicolumn{3}{c|}{ Variables de respuesta en el óptimo } \\
\cline { 2 - 12 } & $\begin{array}{c}\text { Red. } \\
\text { DQO: } d_{1}\end{array}$ & $\begin{array}{c}\text { Red. } \\
\text { Color: } d_{2}\end{array}$ & $\begin{array}{c}\text { Costo Op.: } \\
\mathrm{d}_{3}\end{array}$ & $\mathrm{pH}$ & $\begin{array}{c}{[\mathrm{Fe}]} \\
(\mathrm{mg} / \mathrm{l})\end{array}$ & $\begin{array}{c}{\left[\mathrm{H}_{2} \mathrm{O}_{2}\right]} \\
(\mathrm{mg} / \mathrm{l})\end{array}$ & $\mathrm{DG}$ & $\begin{array}{c}\text { Red DQO } \\
(\%)\end{array}$ & $\begin{array}{c}\text { Red Color } \\
(\%)\end{array}$ & $\begin{array}{c}\text { Costo OP. } \\
\left(\mathrm{USD} / \mathrm{m}^{3}\right)\end{array}$ \\
\hline 1 & $1 / 3$ & $1 / 3$ & $1 / 3$ & 2.70 & 250.2 & 4080 & 0.4961 & 62.42 & 70.90 & 5.465 \\
\hline 2 & 0 & $1 / 2$ & $1 / 2$ & 2.02 & 106.4 & 3105 & 0.5697 & 53.36 & 62.05 & 4.109 \\
\hline 3 & $1 / 2$ & 0 & $1 / 2$ & 3.05 & 274.1 & 3278 & 0.5226 & 57.43 & 59.67 & 4.433 \\
\hline 4 & $1 / 2$ & $1 / 2$ & 0 & 2.38 & 285.0 & 6000 & 0.9898 & 73.95 & 84.69 & 7.986 \\
\hline 5 & $1 / 2-1 / 2 \mathrm{~W}_{3}{ }^{*}$ & $1 / 2-1 / 2 \mathrm{~W}_{3}{ }^{*}$ & $\mathrm{w}_{3}{ }^{*}$ & 2.96 & 331.5 & 5237 & 0.6465 & 71.66 & 79.50 & 7.019 \\
\hline
\end{tabular}

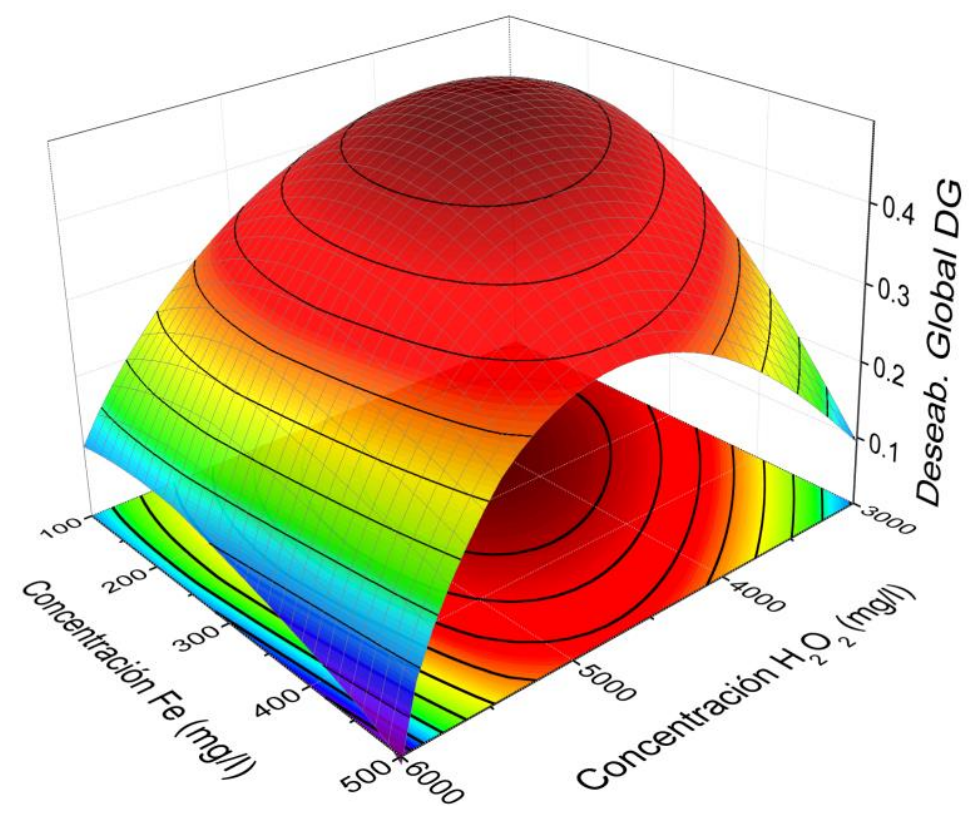

Fig. 3: Cambio de la deseabilidad global con la concentración de hierro y peróxido a un $\mathrm{pH}$ de 2,7 (Factores de peso iguales a 1/3)

Los resultados del análisis económico del este tratamiento se pueden comparar con los obtenidos previamente (Ibarra-Taquez et al., 2017) en donde se obtuvo un costo operacional de $11.9 \mathrm{USD} / \mathrm{m}^{3}$ cuando el tratamiento completo se llevó a cabo utilizando únicamente un proceso de oxidación electroquímica o un costo de $8.9 \mathrm{USD} / \mathrm{m}^{3}$ (utilizando un esquema de electrocoagulación y oxidación electroquímica para cumplir los estándares de la legislación colombiana para el mismo tipo de efluente). Según lo anterior, los costos de operación para el tratamiento por el proceso Fenton son más económicos que cuando se utilizan otros PAOs. Este hecho generalmente ocurre con otro tipo de aguas, por ejemplo para el tratamiento de clorofenol el costo 
utilizando un proceso Fenton es cerca de $8 \mathrm{USD} / \mathrm{m}^{3}$ mientras que usando tecnologías como oxidación electroquímica u ozonización el costo puede llegar a 15 y $81 \mathrm{USD} / \mathrm{m}^{3}$, respectivamente, para iguales niveles de descontaminación (Cañizares et al., 2009). El costo de tratamiento por tecnologías Fenton también está relacionado con la complejidad de las aguas a tratar y puede alcanzar valores tan altos como $60 \mathrm{USD} / \mathrm{m}^{3} \mathrm{si}$ la carga de contaminación es considerablemente alta o si las sustancias en solución son altamente recalcitrantes (Módenes et al., 2012).

\section{CONCLUSIONES}

El tratamiento de aguas residuales provenientes de la industria de café soluble mediante el proceso Fenton permite alcanzar los límites de concentración de DQO exigidos por la legislación colombiana. En este trabajo se estableció una zona de operación bajo la cual se cumple esa normativa ambiental en cuanto a DQO. Dentro de esa zona, la más alta reducción de DQO correspondiente a $80.5 \%$ se alcanzó a un pH de 3.73, a una concentración de hierro igual a $500 \mathrm{mg} / \mathrm{l}$ y a una concentración de peróxido igual a $6000 \mathrm{mg} / \mathrm{l}$. La más alta reducción de color, con un valor de $85.3 \%$, se alcanzó a la misma concentración de peróxido pero a 281.5 $\mathrm{mg} / \mathrm{l}$ de hierro y un $\mathrm{pH}$ de 2.8 debido a la coloración generada por los complejos de hierro con las aguas residuales a altas concentraciones de hierro. Bajo ambas condiciones de operación los costos operacionales fueron altos. Se encontró también que a condiciones de pH igual a 3, concentración de hierro de $331 \mathrm{mg} / \mathrm{l}$ y concentración de peróxido de $5237 \mathrm{mg} / \mathrm{l}$ se obtiene un proceso que cumple los requisitos de ley en cuanto a concentración de DQO con alta decoloración y los más bajos costo operacionales posibles. Para obtener aun menores costos operacionales, el proceso Fenton se podría complementar con otras tecnologías de tratamiento. Las condiciones de operación del proceso Fenton podrían adaptarse de acuerdo a los niveles de eficiencia de la tecnología a la cual se va a acoplar el proceso.

\section{AGRADECIMIENTOS}

Este trabajo se ha desarrollado con el apoyo económico de Colciencias y su Convocatoria Nacional 567 para estudios de Doctorado en Colombia año 2011. Los autores agradecen a Santiago Zuluaga Botero por su participación en el desarrollo de los experimentos.

\section{REFERENCIAS}

Adhoum, N. y L. Monser, Decolourization and Removal of Phenolic Compounds from Olive Mill Wastewater by Electrocoagulation, doi:10.1016/j.cep.2003.12.001, Chem. Eng. Process. Process Intensify, 43, 1281-1287 (2004)

APHA, Standard Methods for the Examination of Water and Wastewater, 21ra Ed., Centennial Edition of American Public Health Association, Washington, USA (2005)

Ay, F., E.C. Catalkaya y F. Kargi, A Statistical Experiment Design Approach for Advanced Oxidation of Direct Red Azodye by Photo-Fenton Treatment, doi:10.1016/j.jhazmat.2008.05.027, J. Hazard. Mater, 162, 230-236 (2009)

Babuponnusami, A. y K. Muthukumar, A Review on Fenton and Improvements to the Fenton Process for Wastewater Treatment, doi:10.1016/j.jece.2013.10.011, J. Environ. Chem. Eng., 2, 557-572 (2014)

Bagal, M. V. y P.R. Gogate, Wastewater Treatment using Hybrid Treatment Schemes based on Cavitation and Fenton Chemistry: a Review, doi:10.1016/j.ultsonch.2013.07.009, Ultrason. Sonochem., 21, 1-14 (2014)

Bianco, B., I. De Michelis y F. Vegliò, Fenton Treatment of Complex Industrial Wastewater: Optimization of Process Conditions by Surface Response Method, doi:10.1016/j.jhazmat.2010.12.054, J. Hazard. Mater., 186, 1733-1738 (2011)

Cañizares, P. y otros tres autores, Costs of the Electrochemical Oxidation of Wastewaters: A Comparison with Ozonation and Fenton Oxidation Processes, doi:10.1016/j.jenvman.2007.10.010, J. Environ. Manage., 90, 410-420 (2009)

Cardenas, A. y otros tres autores, Electrochemical Oxidation of Wastewaters from the Instant Coffee Industry Using a Dimensionally Stable RulrCoOx Anode. ECS Trans., 20, 291-299 (2009)

Clarke, R. y O.G. Vitzthum, Coffee: Recent Developments, ISBN: 978-0-632-05553-1, $1^{\text {a }}$ Ed., 8-12, John Wiley \& Sons, World Agriculture Series, Londres, Inglaterra (2001)

Colombo, R. y otros cuatro autores, Application of the Response Surface and Desirability Design to the Lambda-cyhalothrin Degradation using Photo-Fenton Reaction, doi:10.1016/j.jenvman.2012.12.035, J. Environ. Manage., 118, 32-39 (2013)

Dinsdale, R.M., F. R. Hawkes,y D.L. Hawkes, Mesophilic and Thermophilic Anaerobic Digestion with Thermophilic Preacidification of Instant-Coffee-Production Wastewater, doi:10.1016/S0043-1354(97)00041-9, Water Res., 31, 19311938 (1997)

Farah, A., Coffee Constituents, in: Coffee: Emerging Health Effects and Disease Prevention, doi:10.1002/9781119949893.ch2, 21-58, Wiley-Blackwell, Oxford, Inglaterra (2012)

Fernandez, N. y C.F. Forster, A Study of the Operation of Mesophilic and Thermophilic Anaerobic Filters Treating a Synthetic Coffee Waste, doi:10.1016/0960-8524(93)90115-R, Bioresour. Technol., 45, 223-227 (1993) 
Ferreira, S.L.C y otros diez autores, Box-Behnken Design: An Alternative for the Optimization of Analytical Methods, doi:10.1016/j.aca.2007.07.011, Anal. Chim. Acta, 597, 179-186 (2007)

Foreign Agricultural Service, Coffee: World Markets and Trade (2017)

GilPavas, E. y otros tres autores, Optimización de los Costos de Operación del Proceso de Electro-oxidación para una Planta de Tratamiento de Aguas Mediante Análisis Estadístico de Superficie de Respuesta, doi:10.4067/S071807642016000400008, Inf. Tecnol., 27, 73-82 (2016a)

Gilpavas, E. y otros tres autores, Degradación de Colorante Amarillo 12 de Aguas Residuales Industriales utilizando Hierro Cero Valente, Peróxido de Hidrógeno y Radiación Ultravioleta, doi:10.4067/S0718-07642016000300004, Inf. Tecnol., 27, 23-34 (2016b)

Guedes, A.M. y otros tres autores, Fenton Oxidation of Cork Cooking Wastewater - Overall Kinetic Analysis, doi:10.1016/S0043-1354(03)00178-7, Water Res., 37, 3061-3069 (2003)

Gutierrez-Pulido, H. y R. De la Vara, Análisis y Diseño de Experimentos, ISBN-10: 9701065263, 2da Ed., 351-357, McGrawHill Interamericana Editores S.A, Ciudad de México, México (2008)

Ibarra-Taquez, H.N y otros cuatro autores, Integrated Electrocoagulation-Electrooxidation Process for the Treatment of Soluble Coffee Effluent: Optimization of COD Degradation and Operation Time Analysis, doi:10.1016/j.jenvman.2017.05.095, J. Environ. Manage., 200, 530-538 (2017)

Kušić, $\mathrm{H}$ y otros tres autores, Fenton Type Processes for Minimization of Organic Content in Coloured Wastewaters. Part II: Combination with Zeolites, doi:10.1016/j.dyepig.2006.01.050, Dye. Pigment., 74, 388-395 (2007)

Ministerio de Ambiente y Desarrollo Sostenible de Colombia, Resolución No.0631 de 2015, Artículo 12, (en línea: https://goo.gl/bZQgBk). Acceso: 19 de diciembre (2017)

Módenes, A.N. y otros tres autores, Performance Evaluation of an Integrated Photo-Fenton - Electrocoagulation Process Applied to Pollutant Removal from Tannery Effluent in Batch System, doi:10.1016/j.cej.2012.05.015, Chem. Eng. J., 197, $1-9$ (2012)

Montgomery, D.C., Design and Analysis of Experiments, 8ª Ed., 506-512, John Wiley \& Sons, Inc., Hoboken, USA (2012)

Oller, I., S. Malato y J. Sánchez-Pérez, Combination of Advanced Oxidation Processes and Biological Treatments for Wastewater Decontamination--a Review, doi:10.1016/j.scitotenv.2010.08.061, Sci. Total Environ., 409, 4141-66 (2011)

Panchangam, S.C. y K. Janakiraman, Decolorization of Aqueous Coffee and Tea Infusions by Chemical Coagulation, doi:10.1080/19443994.2013.860401, Desalin. Water Treat., 53, 119-125 (2015)

Pignatello, J.J., E., Oliveros y A. MacKay, Advanced Oxidation Processes for Organic Contaminant Destruction based on the Fenton Reaction and Related Chemistry, doi:10.1080/10643380500326564, Crit. Rev. Environ. Sci. Technol., 36, 1$84(2006)$

Prazeres, A.R., F. Carvalho y J. Rivas, Fenton-like Application to Pretreated Cheese Whey Wastewater, doi:10.1016/j.jenvman.2013.07.016, J. Environ. Manage., 129, 199-205 (2013)

Shoup, M.E., Premiumization Leads the way in Global Coffee Market, including Instant Coffee Category (en línea: https://goo.gl/QbEf47), acceso: 23 de enero (2018)

Tokumura, M. y otros tres autores, UV light Assisted Decolorization of Dark Brown Colored Coffee Effluent by PhotoFenton Reaction, doi:10.1016/j.watres.2006.08.012, Water Res., 40, 3775-84 (2006)

Tokumura, M., H.T. Znad e Y. Kawase, Decolorization of Dark Brown Colored Coffee Effluent by Solar Photo-Fenton Reaction: Effect of Solar Light Dose on Decolorization Kinetics, doi:10.1016/j.watres.2008.08.007, Water Res., 42, 4665$73(2008)$

USP Technologies, Permanganate Titration (en línea: https://goo.gl/fcHAut). Acceso: 10 de diciembre (2017)

Villanueva-Rodríguez, M. y otros cuatro autores, Discoloration and Organic Matter Removal from Coffee Wastewater by Electrochemical Advanced Oxidation Processes, doi:10.1007/s11270-014-2204-6, Water, Air, Soil Pollut., 225, 2204-2215 (2014)

Wang, N. y otros tres autores, A Review on Fenton-like Processes for Organic Wastewater Treatment, doi:10.1016/j.jece.2015.12.016, J. Environ. Chem. Eng., 4, 762-787 (2016)

Zayas Pérez, T., G. Geissler y F. Hernandez, Chemical Oxygen Demand Reduction in Coffee Wastewater through Chemical Flocculation and Advanced Oxidation Processes, J. Environ. Sci. (China), 19, 300-5 (2007) 
